\title{
EVALUATION OF MID-LEVEL MANAGEMENT TRAINING IN IMMUNISATION IN THE AFRICAN REGION
}

\author{
Mutabaruka $E^{1}$, Dochez $C^{2}$, Nshimirimana $D^{1}$, Meheus A. ${ }^{2}$
}

\begin{abstract}
Objective: The Mid-Level Management (MLM) training course provides managers of immunisation programmes with new, advanced skills in planning, management, monitoring and evaluation. An evaluation was conducted of the MLM training courses held between 2000 and 2004 in the African Region, in order to assess its effectiveness and impact, and its contribution to the management of the Extended Programme on Immunisation (EPI) at country level.

Methods: Evaluation methods included: a desk review of the MLM course reports, WHO/AFRO MLM modules and reference documents; interviews with MLM course participants, facilitators, supervisors, Ministry of Health officials and country-based partners; focus group discussions; and questionnaires.

Results: During 2000-2004, eleven MLM courses were held and 642 participants were trained. Of the 151 course participants interviewed, $85 \%$ rated the course as very useful and $15 \%$ as useful. Modules on new vaccines, immunisation safety, cold chain and vaccine management, communication and problem solving were most appreciated. According to supervisors, the MLM training has contributed to significant improvements in the performance of the staff after attending the MLM course. Using DTP3 as an indicator, immunisation coverage in the African Region increased from 49\% in 1991 to $53 \%$ in 2001 and $69 \%$ in 2004.

Conclusions: The MLM training has increased the performance of the trained staff and therefore contributed to the improvement of EPI coverage in the African Region. However, MLM training remains a predominantly vertical event and should be harmonised with other health training programmes for various levels of the health system.
\end{abstract}

Key words: WHO/AFRO, Extended Programme on Immunisation, pre-service training, in-service training, immunisation coverage

\section{Introduction}

The African Region continues to bear a disproportionate burden of vaccine preventable diseases (1). Evidence from various health facility surveys and EPI reviews conducted during the past decade at country and regional levels, shows that the most important barriers to reaching every child in every district with immunisation services are related to planning and management of human, material and financial resources at district and service delivery levels, rather than just physical barriers to access. To overcome these barriers, capacity building is the main operational strategy to improve managerial skills and to integrate the immunisation services within the social and health infrastructure. Therefore, all EPI managers are expected to have practical management skills.

The Mid-Level Management (MLM) training is a two-week intensive course providing managers of immunisation programmes at national, regional and district level with new, advanced skills in planning, management, monitoring and evaluation (2). From the 1980s until 1995, EPI training in the African Region was provided vertically and ad hoc, to meet immediate needs. The training during this period was neither integrated nor comprehensive. The MLM course was originally designed by $\mathrm{WHO} /$ Headquarters during the Universal Child Immunisation Initiative (1985-1990), and it contributed to improvements in programme management and quality. However, with time the content of the training course became outdated, and there were no training materials tailored to the regional needs or adapted to the

Corresponding author: Carine Dochez, tel. +32 3 8202891; Fax: +32 3 8202875; email: carine.dochez@ua.ac.be

${ }^{1}$ World Health Organization Regional Office for Africa, Division of Communicable Diseases, Immunization and Vaccine Development, Brazzaville, Congo, ${ }^{2}$ University of Antwerpen, Department of Epidemiology and Social Medicine, Network for Education and Support in Immunisation, Belgium socio-cultural context of the countries. In addition, external support and resources to the programme diminished and the MLM training frequency dropped considerably. Between 1994 and 1999 no MLM courses were organised in the African Region.

In order to enhance the performance of national immunisation programmes, Training Needs Assessments (TNAs) conducted in 12 target countries identified the need for management training (3). It also identified bottlenecks between pre- and in-service training, poor coverage of EPI topics in the curricula of health schools and lack of training in EPI of teachers. This situation has necessitated the broader collaborative efforts from partners (WHO, UNICEF, USAID, UNF (United Nations Foundation), NESI (Network for Education and Support in Immunisation) and CVP/PATH (Children's Vaccine Program of PATH)) to revive the MLM training. Significant progress in EPI mid-level management training at inter-country and country levels has been made since 2000, through training materials development and running of various MLM courses. From 2000 to 2004, eleven inter-country MLM courses were held in the following locations: Niamey (2000); Abuja and Douala (2001); Maputo, Windhoek and Dakar (2002); Pretoria and Benin (2003); Cape Town, Dakar and Maputo (2004).

A summative evaluation (4) has been carried out to highlight the role of MLM training in the improvement of the immunisation programme management in the African Region. The objectives of the evaluation were to assess the effectiveness and the impact of inter-country and national MLM training courses (2000-2004) and its contribution to the management of EPI services at country level.

\section{Methods}

The MLM training evaluation was based on a protocol developed by VPD/AFRO (Vaccine Preventable Diseases unit of WHO/AFRO). The initial, internal evaluation was carried out through a desk review of 
MLM training data at the Inter-country and Regional offices. The internal evaluation team was represented by VPD/AFRO staff, the EPI capacity building officer, WHO/AFRO inter-country staff and a consultant. The second, external phase of the evaluation was conducted by a multidisciplinary team composed of experts from WHO, UNICEF, UNF, USAID and NESI. The external evaluation was conducted at the WHO/AFRO Regional Office and in five selected countries (Ethiopia, Ghana, Lesotho, Senegal and Zambia).

The methods of the evaluation included:

- Desk review of the information on inter-country and in-country EPI MLM courses held during 2000-2004;

- Review of curricula at regional and country levels;

- Review of WHO/AFRO MLM modules, related reference documents and handouts;

- Country visits for qualitative data collection and analysis:

○ Interviews with past MLM course participants, facilitators, supervisors, Ministry of Health officials, countrybased partners and other stakeholders;

- Focus group discussions with past course participants and facilitators;

- Questionnaires sent to past MLM course participants and facilitators.

\section{Results}

\section{Results within pre-service training institutions}

The evaluation team visited 26 pre-service training institutions in the five selected countries and had interviews with the principals and the teaching staff. These visits revealed inadequacies both in theoretical content and practical exposure to immunisation, as well as in availability of relevant training materials, tools and reference materials. These observations were in line with findings of Training Needs Assessments in other countries of the African Region (3). Only 27\% of the training institutions reviewed mentioned that the curriculum is adequate for EPI teaching. Less than 20\% of training schools had sufficient teaching materials, reference books and access to EPI publications (Figure $1)$.

\section{Satisfactory index on the content and overall assessment of the individual MLM modules}

The participants evaluated the content of each module and gave their overall satisfaction with the course. Figure 2 shows that the average scores for the content of all modules and overall course assessment exceeded the threshold of $70 \%$ satisfaction.

Participants indicated their overall satisfaction with the MLM course by responding whether the course was "Very useful", "Useful", "Somewhat useful" or "Not useful". Of the 151 course participants interviewed, $85 \%$ rated the course as very useful and $15 \%$ as useful.
Various components of the MLM course were assessed by the course participants: course materials, exercises, plenaries, group discussions, individual discussions with facilitators, course facilitation as well as administrative arrangements. Figure 3 shows that all components of the course were assessed as "Very useful" or "Useful" by most of the participants. The high proportion of "Not useful" responses to the question on individual discussions with facilitators were from Lesotho (10 out of 23 respondents) and Zambia (7 out of 30 respondents). Because of the shorter period of the MLM courses in those two countries, there was not sufficient time for individual discussions.

\section{Focus group discussions with participants}

The external evaluation teams held eight focus group discussions with participants from various MLM courses in the five selected countries.

Participants found the MLM course very relevant or relevant to their actual work because of the following reasons:

- It updated them on new developments in EPI and helped them with their current job, filling the knowledge gap in EPI;

- It enhanced their performance in data management and analysis;

- It offered a more comprehensive and systematic way of implementing EPI;

- It facilitated harmonisation of theory taught in training schools with field practice;

- It helped them to train other health workers;

- Work of supervising became easier due to better understanding of various aspects of the programme.

Modules on new vaccines, immunisation safety, cold chain and vaccine management, communication and problem solving were most appreciated by participants.

The lack of site visits and a tight time schedule (e.g. not enough time for group discussions, some exercises and individual discussions with facilitators) were identified as weak points of the MLM training.

\section{Profile of Participants Trained at AFRO Inter- country MLM Courses}

Out of 642 participants trained, $416(65 \%)$ were EPI managers from national, provincial and district levels. Figure 4 shows that $31 \%$ (199) of the participants were EPI managers from sub-national level (provinces and districts). The proportion of country based WHO and UNICEF participants reached $18 \%$, indicating high commitment of these partners to immunisation programmes. An increase in training of academic staff occurred during the last five courses: 110 professors/teachers from universities and nursing schools were trained in MLM. After returning to their respective institutions, they introduced changes in EPI teaching based on new developments in the immunisation programme. This is an investment towards pre- and inservice training.

\section{Assessment of performance}


About $25 \%$ of participants were promoted in post or advanced in their career after following the MLM training course (Table 1). Although promotions in some countries were related to the years of service, participation in and skills development from courses like the MLM training were taken into consideration when competing candidates applied for higher posts.

Table 1: Career advancement of participants after MLM training

$\begin{array}{lcccc}\begin{array}{l}\text { Countries } \\ \text { visited }\end{array} & \begin{array}{l}\text { Number } \\ \text { interviewed }\end{array} & \begin{array}{l}\text { Same } \\ \text { position } \\ \text { after } \\ \text { MLM } \\ \text { training }\end{array} & \begin{array}{l}\text { Advanced } \\ \text { in career } \\ \text { after } \\ \text { MLM } \\ \text { training }\end{array} & \begin{array}{l}\text { Percentage } \\ (\%)\end{array} \\ \text { Ethiopia } & 22 & 12 & 10 & 45.5 \\ \text { Ghana } & 51 & 37 & 14 & 27.5 \\ \text { Lesotho } & 23 & 17 & 6 & 26.0 \\ \text { Senegal } & 26 & 23 & 3 & 11.6 \\ \text { Zambia } & 30 & 25 & 5 & 17.0 \\ \text { Total } & 152 & 114 & 38 & 25.0\end{array}$

\section{Assessment of trainees by supervisors}

To obtain supervisors' views on participants' performance prior and after MLM training, interviews were held with immediate supervisors of 42 past participants. Questions were addressed to supervisors on various performance categories, asking them to score answers using " 3 " for very good or excellent performance, " 2 " for good or satisfactory performance and " 1 " for below average performance. The results of this interview were expressed as a value of the mean score per response prior and after participant's MLM training (Figure 5). According to supervisors, the MLM training has contributed to significant improvements in the performance of the MLM participants (Figure 5). The most profound changes were in the area of technical competence and updated EPI knowledge, management, planning, and partner coordination skills. There was also perceived benefit of the training for the supervisors themselves, as they could now share important responsibilities and workload with the newly trained staff.

\section{Facilitators' Profile}

WHO/AFRO and WHO inter-country teams facilitated in the eleven MLM inter-country courses. A large group of facilitators were from the respective Ministries of Health, and a smaller group were lecturers from training institutions. Other facilitators were from the UNICEF Regional Offices, WHO country based focal points, WHO Headquarters, NESI, AMP and $\mathrm{CVP} / \mathrm{PATH}$. It is important to have co-facilitators among the participants, as on their return to their respective countries, these co-facilitators can organise national MLM courses using the experience in facilitation gained, and as such create a critical mass of facilitators at country level.

\section{Impact of MLM Training}

Among the recommended actions of the African Regional EPI Strategic Plan 2001-2005 (5), systematic evaluation was proposed to assess progress made in training and its impact on the reduction of morbidity and mortality of vaccine preventable diseases.

It is, however, recognized that training accountability and impact is difficult to measure, as indicators of training quality are ad hoc. Some of the indirect indicators may provide information on long-term benefits of training in management, e.g.:

- frequency of occurrence of target disease outbreaks in catchment areas where staff were trained compared with other areas with low training activity;

- AEFI incidence in catchment areas where staff were trained;

- target diseases incidence in catchment areas where staff were trained compared with other areas with low training activity;

- Immunisation coverage in catchment areas where staff were trained.

To test the last indicator, the trend in immunisation coverage (using DTP3 as an indicator) in five countries was compared between training stagnation period (19961999) and training activity period (2000-2004), indicating positive changes in the national DTP3 coverage during 2000-2004 (Figure 6).

Improvements in immunisation programme indicators including vaccination coverage cannot be contributed solely to training. However, through perception of users and country based partners, satisfaction index results and observations in the field during the external evaluation, one can conclude that the MLM training has increased the performance of the trained staff and therefore contributed to the improvement of EPI coverage in the African Region. This was also confirmed through the evaluation of Reaching Every District (RED) (6).

\section{MLM Training Sustainability - National Commitment and Partner Support}

The immunisation programmes in the African Region continue to rely on government support and external funding. National governments contribute substantial money and non-monetary resources for the implementation of immunisation activities including training. Tremendous commitment has been made by GAVI partners towards supporting immunisation programmes in the African Region with an emphasis on strengthening routine immunisation, including training. An immediate result of stronger partnerships was an increase in the level of funding for MLM training activities especially by UNICEF, USAID, UNF and NESI. Partners contributed not only financially but participated also in proof reading, in technical review of the module content as well as facilitation in training activities. 


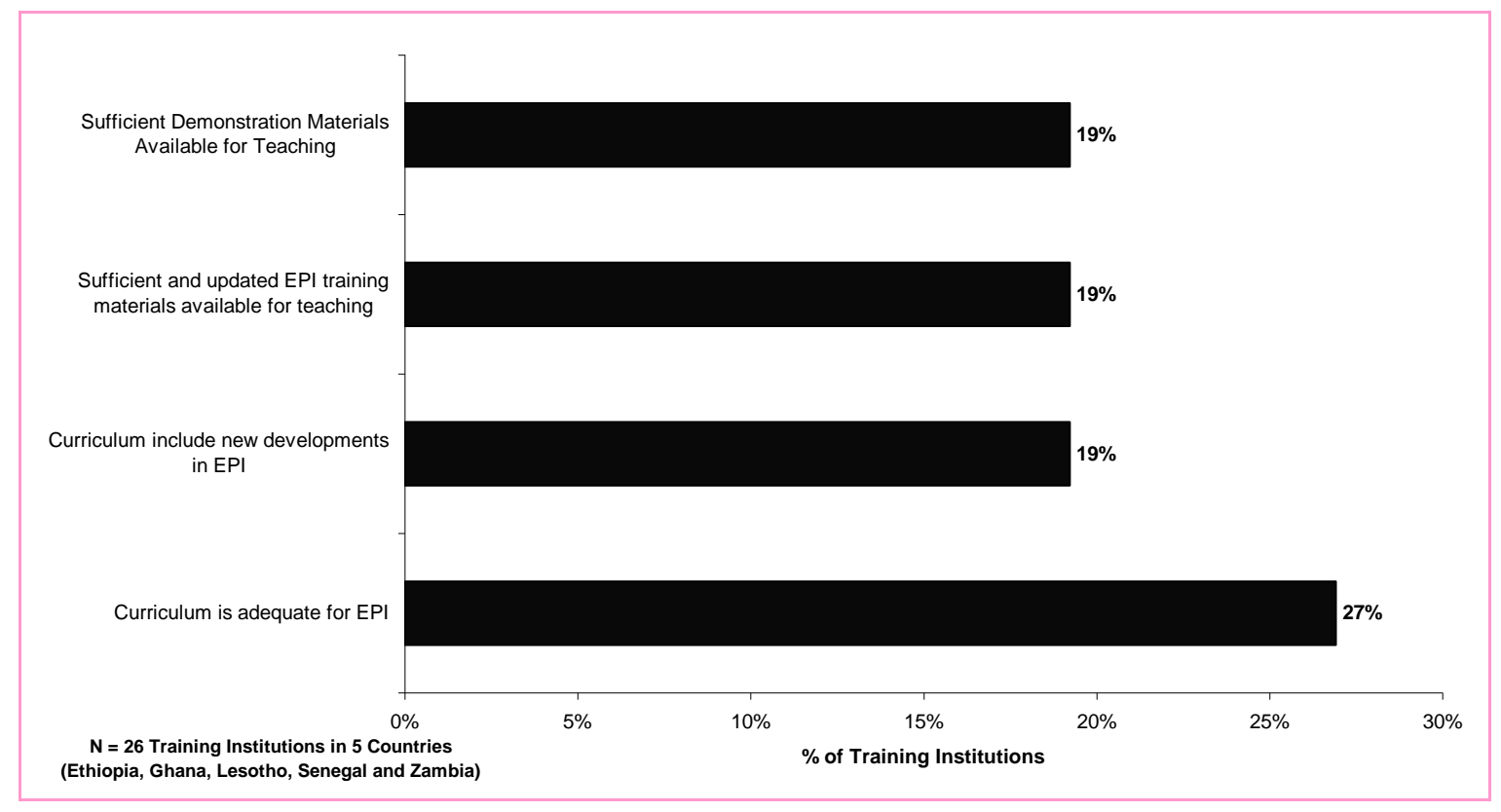

Figure 1: EPI content in curriculum and availability of training materials in 26 training institutions in five selected countries.

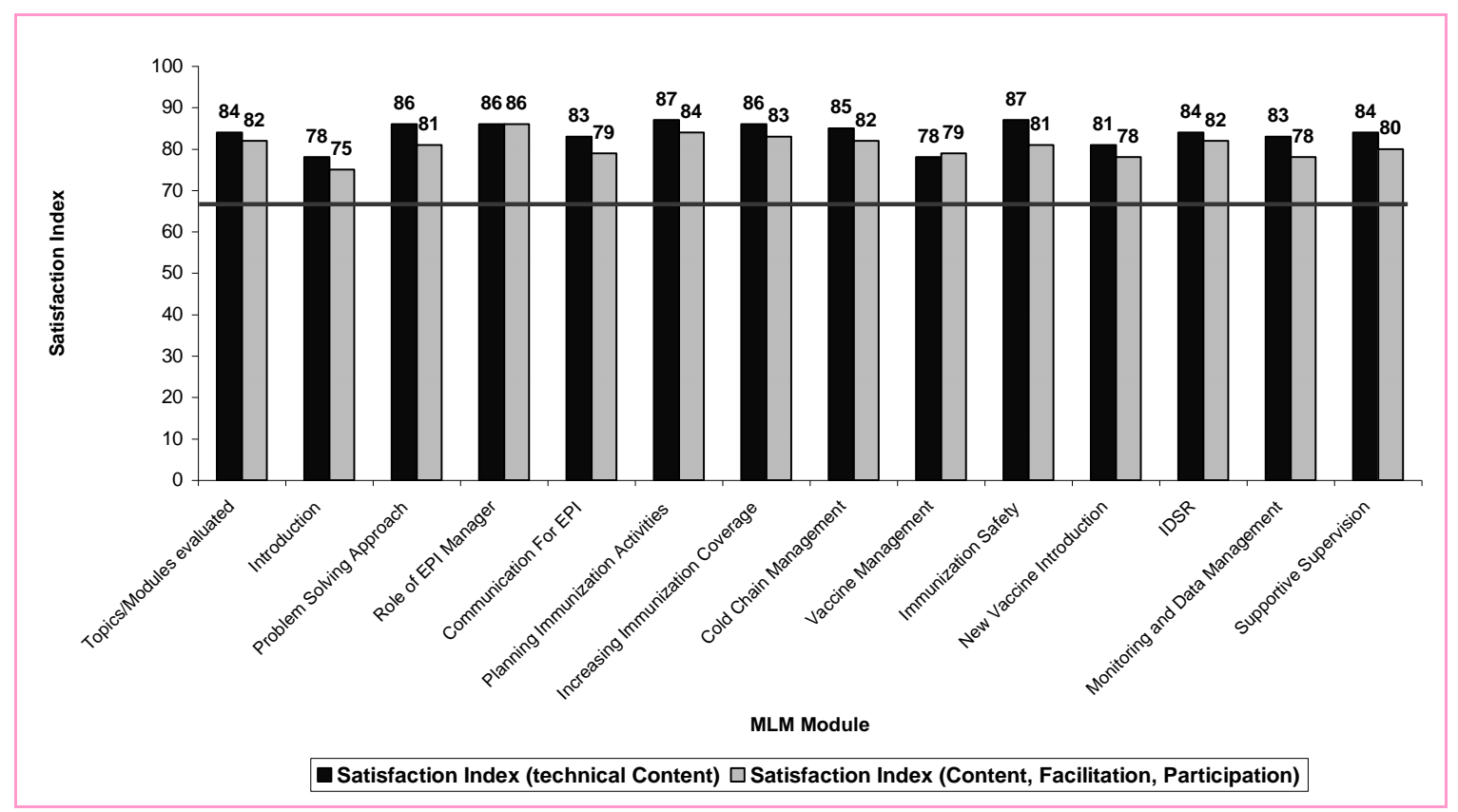

Figure 2: Satisfaction index for course modules at inter-country MLM courses 


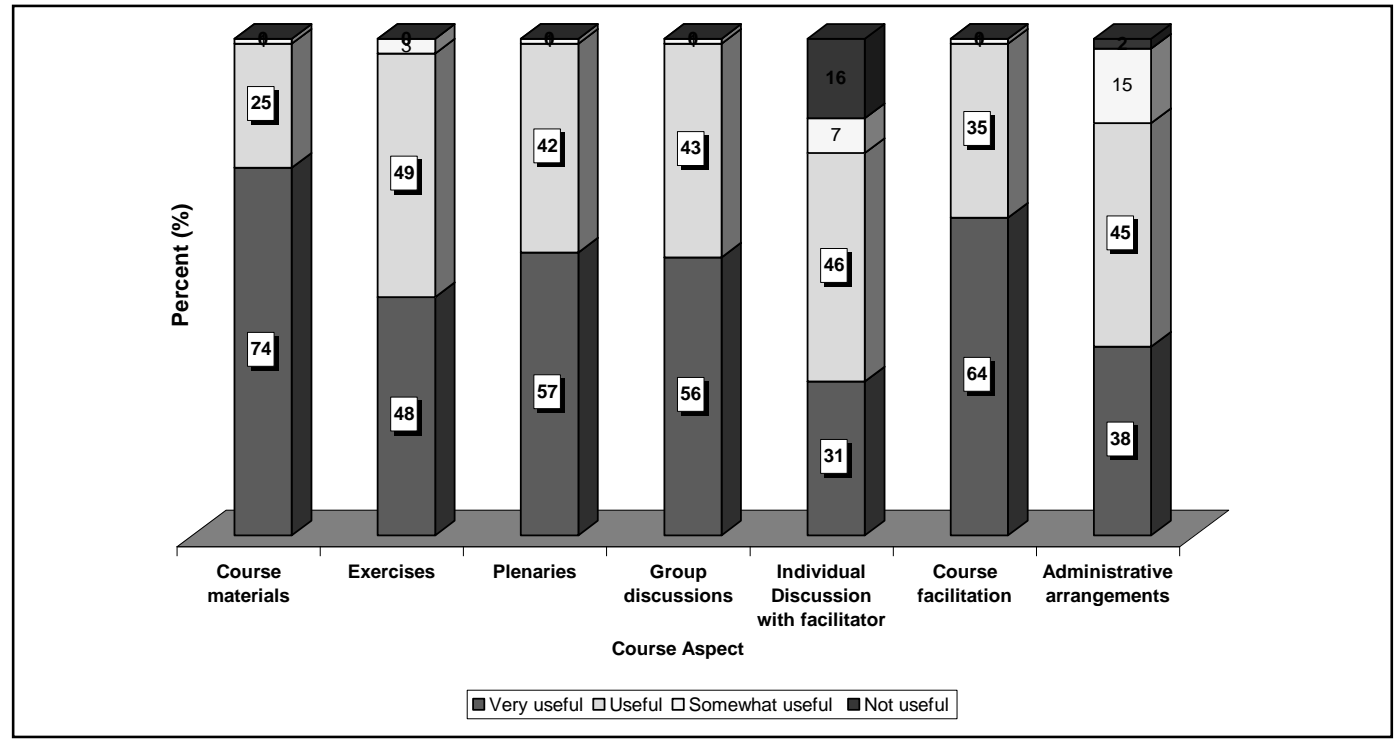

Figure 3. assessmet of various aspects pf mational MLM courses by participants In the five countries (Ehiopian, Ghana. Lesotho, Senegal and zambia) visited

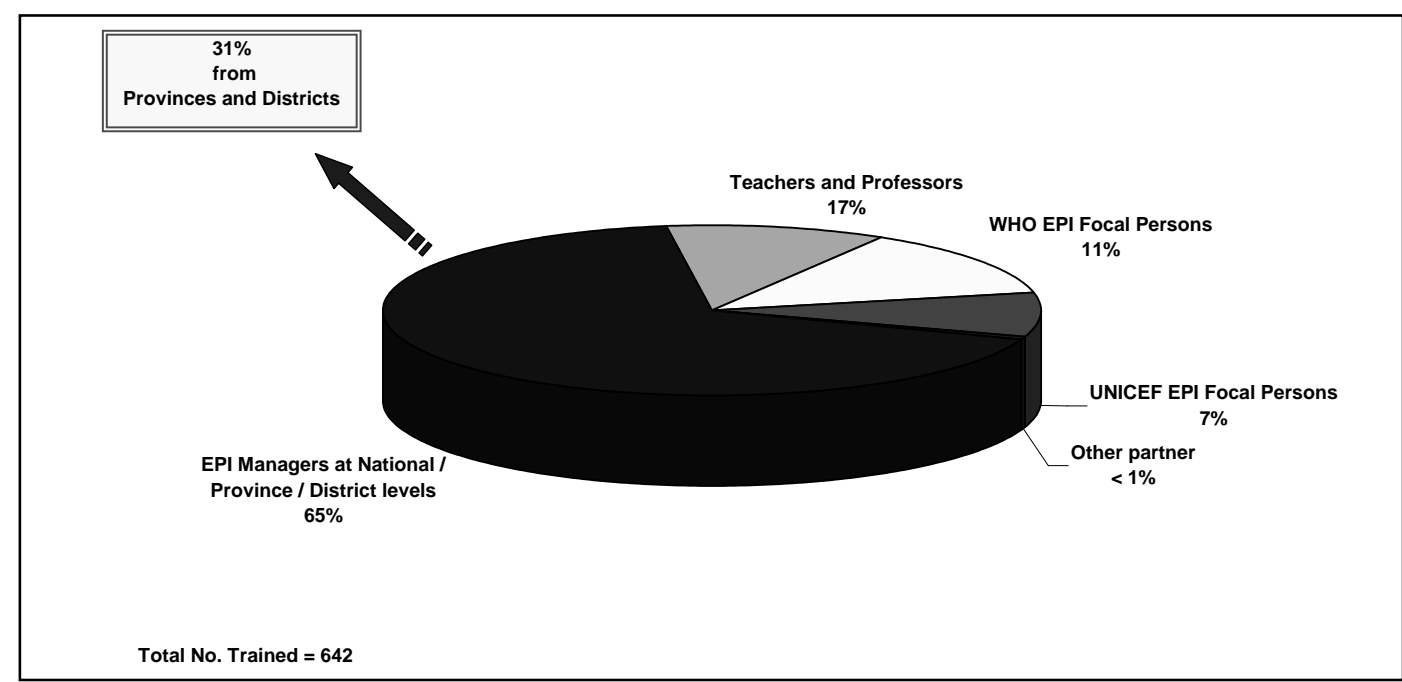

Figure 4: Profile of MLM participants in inter-country MLM courses held from 2000-2004 


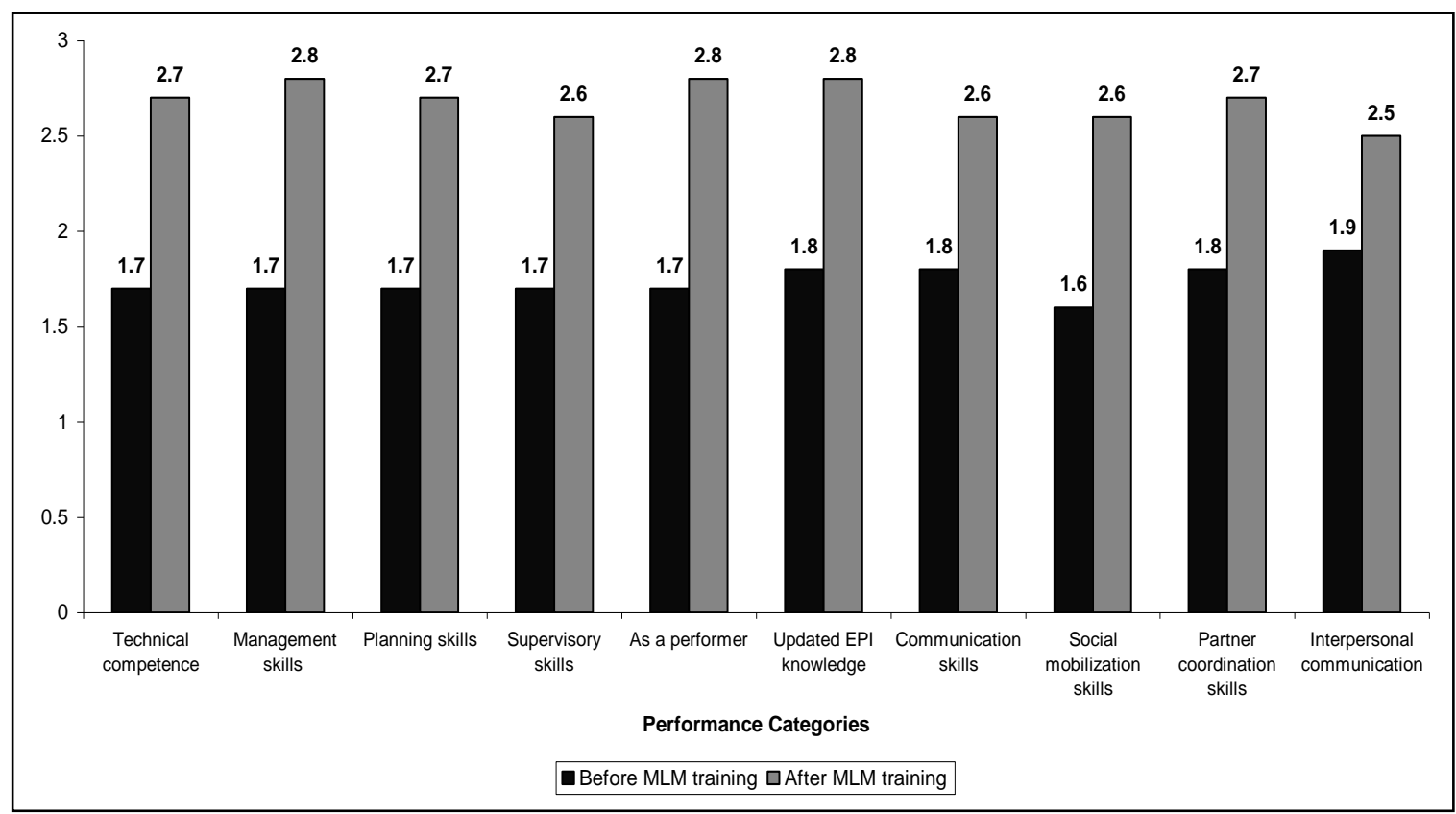

Note: These were mean values adjusted to the number of responses in five countries

Figure 5: Assessment of performance of MLM past participants by their immediate supervisors

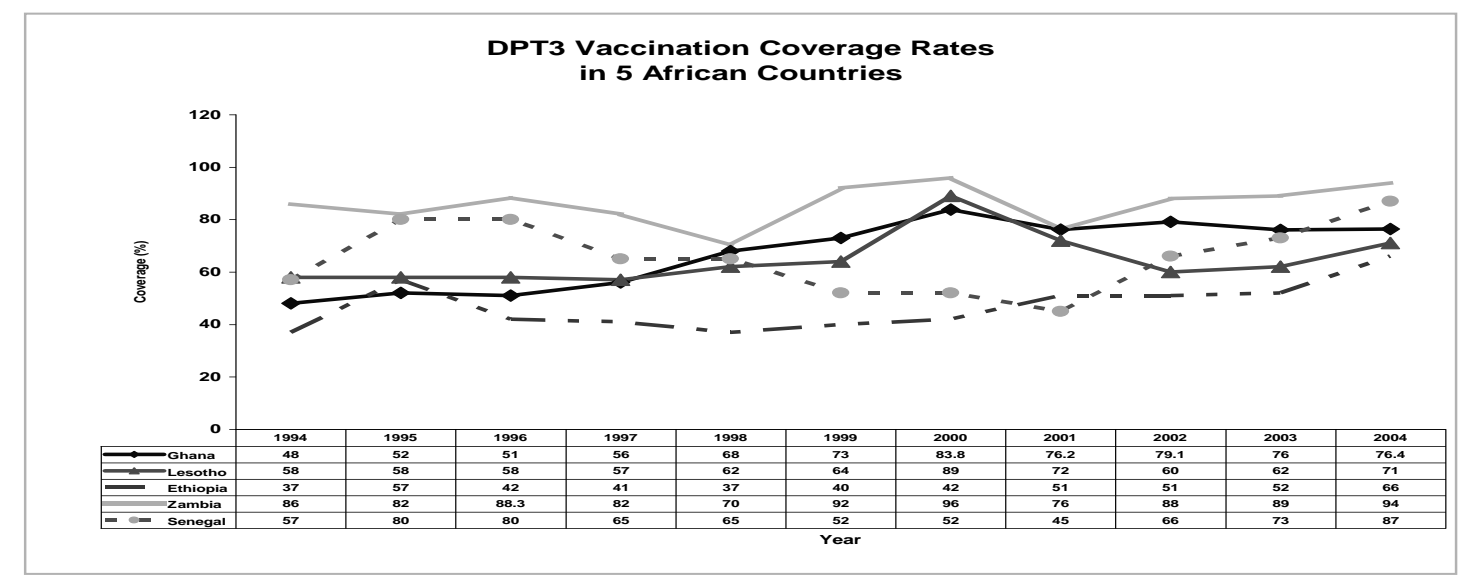

Figure 6: DPT3 coverage trends from 1994 to 2004 in five African countries 


\section{Discussion}

Many countries in the African Region conducted programme reviews and training needs assessments to identify gaps in the management of national immunisation programmes and needs in training to address these gaps. During 2000-2004, eleven intercountry MLM courses were held and 642 participants were trained. Each of the five countries (Ethiopia, Ghana, Lesotho, Senegal and Zambia) visited expanded the MLM training through cascading it to province and district levels, and had a pool of facilitators to run a selfsufficient MLM course.

There is a set of well elaborated, structured and learner-friendly MLM modules developed by WHO/AFRO in collaboration with partners, which have undergone a series of testing and validation by experts and MLM course participants. Some countries adapted these MLM modules to suit their country situation. About half of the countries in the African Region (including the five countries visited by the evaluation team) conducted national MLM courses using the generic or adapted WHO/AFRO MLM course modules to train their national and sub-national EPI managers.

The programme has a tremendous support and involvement of partners who consider MLM training as a joint venture. Country based partners consider MLM training as a "good value for money". The current evaluation exercise was a good example of collaboration, having evaluators from WHO, UNICEF, UNF, USAID and NESI.

Despite practical steps towards programme integration, MLM training remains a predominantly vertical event. Mid-level management training should be harmonised with other health training programmes for various levels of the health system.

A bottleneck was identified between pre-service training and national EPI services due to outdated curriculum at pre-service institutions (results of most TNAs) and current practices of EPI applying innovations and new technologies (e.g. new vaccines, combined vaccines, auto-disable syringes). At country level, there is a lack of training materials in local languages. Consideration should be given to institutionalisation of the MLM course in three public health training centres in the Region for English, French and Portuguese speaking countries. Teaching in EPI needs to be further strengthened in the basic training of health personnel to equip the new graduates with the necessary skills and knowledge in line with new developments, the Global Immunisation Vision and Strategy (GIVS) (7) and the Millennium Development Goals (8). As the immunisation programme is characterized by rapid progression, regular revision and standardisation of the existing guidelines, tools and training materials will be necessary.
An increase in training of academics occurred during the years 2003 and 2004; around 110 academics from universities and nursing colleges were trained in MLM. After returning to their respective institutions, they introduced changes in EPI teaching based on new developments and strategies in EPI (e.g. GIVS, RED, introduction of new vaccines).

The MLM training has increased the performance of the trained staff and therefore contributed to the improvement of EPI coverage in the African Region (DTP3 regional coverage was $49 \%$ in $1991 ; 53 \%$ in 2001 and $69 \%$ in 2004). Similar improvements in DTP3 coverage rates have been observed in the five visited countries. Furthermore, a positive association was found between health worker density and childhood vaccination coverage in developing countries (9).

MLM courses provide a forum to exchange experiences among various countries within the African Region and bring together internationally recognized immunisation experts and national managers to share theory and practice in programme management. It is also a means to update participants and facilitators on the latest technical achievements in the immunisation programme and on recommendations and strategic visions of global and regional bodies (e.g. World Health Assembly, WHO and UNICEF Regional Committee meetings, Task Force on Immunisation, GAVI).

\section{Acknowledgements}

The authors whish to thank Dr. Lev Arevshatian for the compilation of the MLM summative evaluation report.

\section{References}

1. WHO. The global burden of disease: 2004 update. World Health Organisation 2008, Geneva. http://www.who.int/healthinfo/global_burden_disease/GBD_report_2004up date full.pdf

2. WHO/AFRO. Mid-Level management course for EPI managers. WHO/AFRO http://www.afro.who.int/dde/vpd/epi_mang_course/index.html

3. Mutabaruka E, Nshimirimana D, Goilav C, Meheus A. EPI Training Need Assessment in 12 African Countries, 2002 - 2004. WHO Regional Office for Africa. Communicable Diseases Bulletin for the African Region. 2005; 3(2): $1-4$.

4. WHO/AFRO. Mid-level management training in immunisation in the African Region 2000-2004. Summative evaluation. WHO/AFRO 2005.

1. http://www.who.int/immunization_training/reports/MLM\%20course\%20Ev aluation final.pd

5. WHO. EPI Strategic Plan 2001-2005. WHO. http://www.afro.who.int/ddc/vpd/epi mang course/pdfs/english/red.pdf

6. WHO/AFRO. In-depth evaluation of the Reaching Every District approach in the African Region. WHO/AFRO 2007. http://www.afro.who.int/dde/vpd/routine/red-2007.pdf

7. WHO/IVD and UNICEF. Global Immunization Vision and Strategy 2006 2015. WHO and UNICEF 2005. http://www.who.int/vaccinesdocuments/DocsPDF05/GIVS_Final_EN.pdf

8. UN. Millennium Development goals. http://un.org/millenniumgoals/

9. Arnand S, Barnighausen T. Health workers and vaccination coverage in developing countries: an economic analysis. The Lancet 2007; 369: 1277 develo 